\title{
VALOR DA ULTRA-SONOGRAFIA E DA TOMOGRAFIA COMPUTADORIZADA NO TRATAMENTO CIRÚRGICO DAS NEOPLASIAS DA GLÂNDULA PARÓTIDA
}

\section{THE ROLE FOR ULTRASONOGRAPHYAND COMPUTED TOMOGRAPHY IN THE EVALUATION OFTHE SURGICAL TREATMENT OF PAROTID GLAND NEOPLASMS}

\author{
Margarida Maria dos Santos Antunes ${ }^{1}$ \\ Josias de Andrade Sobrinho,ECBC-SP ${ }^{2}$ \\ Ricardo Pires de Souza $^{3}$ \\ Abrão Rapoport, ECBC-SP ${ }^{2}$
}

\begin{abstract}
RESUMO: Objetivo: Avaliar o valor da ultra-sonografia (US) e tomografia computadorizada (TC) no planejamento cirúrgico das neoplasias da glândula parótida. Método: Estudo retrospectivo de 56 pacientes portadores de neoplasias da glândula parótida (43 benignas e 13 malignas), tratados nos Departamentos de Cirurgia de Cabeça e Pescoço/Otorrinolaringologia e Diagnóstico por Imagem do Hospital Heliópolis, Hosphel, São Paulo (1991 a 1999). Comparamos os achados por US (45) e TC (15) com os histopatológicos pós-cirúrgicos empregando-se o teste diagnóstico de sensibilidade e especificidade. Resultados: Dos 45 exames de US, a consistência sólida foi diagnosticada em 33 casos (sensibilidade de $89 \%$ e especificidade de 50\%) e a cística em quatro casos (sensibilidade e especificidade de 50\%). Quanto à localização no lobo superficial, houve coincidência em 31 casos (sensibilidade de $91,2 \%$ e especificidade em 36,5\%), e para o lobo profundo em quatro casos (sensibilidade de $40 \%$ e especificidade de 88,6\%). Quanto aos 15 exames de TC, a consistência sólida foi demonstrada em 13 casos (sensibilidade de 86,7\% e especificidade de $0 \%$ ) e a cística em nenhum caso (sensibilidade de $0 \%$ e especificidade de $86,7 \%$ ). Quanto à localização no lobo superficial, houve coincidência em um caso (sensibilidade de $20 \%$ e especificidade em 90\%), e para o lobo profundo em nove casos (sensibilidade de $90 \%$ e especificidade de $20 \%$ ).Conclusões: 1 . A US foi o método de escolha para lesões sólidas no lobo superficial e falha no lobo profundo. 2. A TC foi o método de escolha para as lesões do lobo profundo, e falhou nas lesões císticas no lobo superficial.
\end{abstract}

Descritores: Ultrasonografia; Tomografia computadorizada; Planejamento de assistência ao paciente; Glândula parótida; Neoplasias; Estudos retrospectivos.

\section{INTRODUÇÃO}

Antes do advento da ultra-sonografia (US) ${ }^{1}$ em 1971 e da Tomografia Computadorizada (TC) ${ }^{2}$ em 1973, as neoplasias benignas e malignas da glândula parótida eram avaliadas através da radiologia convencional e através do exame palpatório feito pelo especialista. Enquanto as lesões do lobo superficial eram facilmente diagnosticadas, as do lobo profundo somente eram detectadas quando ocorria o abaulamento da parede lateral da orofaringe e através do exame de orofaringoscopia. Assim, o advento dos métodos de imagem vieram contribuir com a elucidação da natureza das neoplasias (císticas ou

1. Mestre em Cirurgia de Cabeça e Pescoço, Hospital Heliópolis, Hosphel São Paulo, SP.

2. Cirurgião em Cirurgia de Cabeça e Pescoço/ Otorrinolaringologia, Hospital Heliópolis, Hosphel ,São Paulo, SP.

3. Radiologista do Hospital Heliópolis, Hosphel, São Paulo, SP.

Recebido em 26/07/2002

Aceito para publicação em 17/12/2002

Trabalho realizado pelos Departamentos de Cirurgia de Cabeça e Pescoço/Otorrinolaringologia e Diagnóstico por Imagem do Hospital Heliópolis, Hosphel, São Paulo, SP. 
sólidas) através da US para o lobo superficial, e pela TC para o lobo profundo (predominantemente no prolongamento faríngeo) da glândula parótida.

Fica portanto evidente que para um planejamento terapêutico cirúrgico para as neoplasias da glândula parótida, impõe-se o emprego da imagem para detecção da natureza da doença, bem como para avaliar o comprometimento de um ou mais lobos da glândula, comparando-se o achado pré cirúrgico com o exame anátomo patológico da peça operatória ${ }^{3-8}$.

\section{MÉTODO}

Foram analisados retrospectivamente os prontuários e os exames de diagnóstico por imagem de 56 pacientes do Departamento de Cirurgia de Cabeça e Pescoço e Otorrinolaringologia do Hospital Heliópolis, Hosphel, São Paulo, portadores de lesões nodulares (ou não) de glândula parótida, de 1991 a 1999.

Foram feitas 45 ultra-sonografias (US) e 15 tomografias computadorizadas (TC), totalizando 60 exames, e os resultados comparados com o achado cirúrgico.

Quanto aos exames por imagem empregados, a função da US para a glândula parótida é analisar se a lesão é bem delimitada e inteiramente visibilizada e se sua natureza é cística ou sólida tendo como limitação o lobo profundo, por interposição do ramo da mandíbula.Os exames de US foram realizados no Serviço de Diagnóstico por Imagem do Hospital Heliópolis, em aparelho Sinergy C-B, versão SW 2.3111, GE Medical Systems, General Electric Company, Milwaukee, Wiscousin, EUA, com o emprego de transdutores de alta freqüência ( 7 a $10 \mathrm{MHz}$ ).

A TC determina também a natureza cística ou sólida das lesões, além do acometimento de estruturas adjacentes e invasão óssea. Os exames de TC foram realizados em equipamento de terceira geração, como um Toshiba TCT $500 \mathrm{~S}$, Toshiba Medical do Brasil, Toshiba Corporation, Tokio, Japan, com cortes com espessura e incremento de $5 \mathrm{~mm}$, da base do crânio à fúrcula esternal, durante a administração do meio de contraste endovenoso.

Quanto à metodologia propedêutica-clínica, esta foi empregada através da inspeção e palpação do pescoço e da orofaringe, procurando-se detectar assimetrias, abaulamentos, presença de neoplasias de lobo superficial ou profundo, bem como sinais de comprometimento do nervo facial, e eventuais linfonodos cervicais inflamatórios ou metastáticos.

Quanto ao procedimento cirúrgico, em todos os pacientes estudados, realizou-se parotidectomia parcial para os tumores benignos do lobo superficial e total para os do lobo profundo, bem como total para os tumores malignos com ou sem preservação do nervo facial.

Para a metodologia estatística foram utilizadas estatísticas descritivas média e mediana para a idade dos pacientes, e freqüências relativas e absolutas das variáveis raça, sexo, métodos por imagem (US, TC), procedimentos cirúrgicos e tipos histológicos das neoplasias.

Para a avaliação da acurácia da US e TC na determinação do tipo de tumor (sólido ou cístico) e da localização do mesmo (superficial ou profundo), foram calculados a sensibilidade e a especificidade de ambos os métodos diagnósticos, utilizando como padrão ouro o exame anátomo-patológico.

$$
\begin{aligned}
& \text { Sensibilidade }=\mathrm{VP} / \mathrm{VP}+\mathrm{FN} \times 100 \\
& \text { Especificidade }=\mathrm{VN} / \mathrm{VN}+\mathrm{FP} \times 100 \\
& \mathrm{VP}=\text { verdadeiros positivos } \\
& \mathrm{VN}=\text { verdadeiros negativos } \\
& \mathrm{FN}=\text { falsos negativos } \\
& \mathrm{FP}=\text { falsos positivos }
\end{aligned}
$$

O valor preditivo positivo (VPP) foi utilizado para estimar qual a probabilidade de o tumor realmente ser superficial ou profundo, sólido ou cístico, uma vez que o exame foi positivo (FP). $\mathrm{O}$ valor preditivo negativo (VPN) estimou a probabilidade de o tumor não apresentar uma das características, uma vez que o exame (US ou TC) foi negativo (FN). Ambos os valores foram determinados em taxas segundo fórmulas abaixo.

$$
\begin{aligned}
& \mathrm{VPP}=\mathrm{VP} / \mathrm{VP}+\mathrm{FP} \times 100 \\
& \mathrm{VPN}=\mathrm{VN} / \mathrm{VN}+\mathrm{FN} \times 100
\end{aligned}
$$

\section{RESULTADOS}

Dos 56 casos avaliados, 43 correspondiam a neoplasias benignas e 13 a neoplasias malígnas (Tabelas 1 e 2).

Quanto à localização das neoplasias, 37 (66.1\%) eram de lobo superficial e 17 (33.9\%) do 
Tabela 1 - Distribuição das neoplasias benignas segundo o tipo histológico.

\begin{tabular}{lrr}
\hline NEOPLASIAS BENIGNAS & N & \% \\
\hline Adenoma pleomorfo & 24 & 56 \\
Tumor de Warthin & 11 & 26 \\
Adenoma de células basais & 3 & 7 \\
Lipoma & 2 & 5 \\
Cisto linfoepitelial & 1 & 2 \\
Cisto dermóide & 1 & 2 \\
Cistoadenoma & 1 & 2 \\
TOTAL & $\mathbf{4 3}$ & $\mathbf{1 0 0}$ \\
\hline
\end{tabular}

Tabela 2 - Distribuição das neoplasias malignas segundo o tipo histológico.

\begin{tabular}{lrr}
\hline NEOPLASIAS MALIGNAS & N & \% \\
\hline Adenocarcinoma & 3 & 23 \\
Carcinoma mucoepidermóide & 3 & 23 \\
Carcinoma epidermóide & 2 & 15 \\
Carcinoma adenocístico & 2 & 15 \\
Carcinoma ductal & 1 & 8 \\
Carcinoma de células acinares & 1 & 8 \\
Lipossarcoma & 1 & 8 \\
TOTAL & $\mathbf{1 3}$ & $\mathbf{1 0 0}$ \\
\hline
\end{tabular}

Tabela 3 - Distribuição das lesões segundo os achados macroscópicos e ultrassonográficos.

\begin{tabular}{lcc}
\hline & \multicolumn{2}{c}{ Exame anátomo-patológico } \\
\hline US & Sólido & Cístico \\
Sólido & 33 & 4 \\
Cístico & 4 & 4 \\
\hline
\end{tabular}

profundo. De acordo com o sexo e raça 21 (62.5\%) eram do sexo masculino e $35(37,5 \%)$ do feminino; $41(73.2 \%)$ da raça branca e $15(26.8 \%)$ negra.

Dos 45 exames de ultra-sonografia, a consistência sólida das neoplasias foi demonstrada corretamente em 33 casos, com uma sensibilidade de $89,2 \%$ e especificidade de $50 \%$, e para cística em quatro casos, com sensibilidade e especificidade de $50 \%$ (Tabela 3). Quanto à localização no lobo superficial, demonstrou corretamente em 31 casos, sensibilidade de $91,2 \%$ e especificidade de $36,5 \%$, e para o lobo profundo em quatro casos,
Tabela 4 - Distribuição das lesões segundo os resultados do exame anátomo-patológico e ultrasonografia quanto à localização da lesão (superficial ou não superficial).

\begin{tabular}{lcc}
\hline & \multicolumn{2}{c}{ Localização } \\
\hline US & Superficial & Profunda \\
Superficial & 31 & 6 \\
Profunda & 4 & 4 \\
\hline
\end{tabular}

Tabela 5 - Distribuição das lesões segundo os resultados do exame anátomo-patológico $e$ tomografia computadorizada quanto às características da lesão (sólida ou cística).

\begin{tabular}{lcc}
\hline \multicolumn{3}{c}{ Exame anátomo-patológico } \\
\hline Tomografia computadorizada & Sólida & Cística \\
Sólida & 13 & 0 \\
Cística & 2 & 0 \\
\hline
\end{tabular}

Tabela 6 - Distribuição das lesões segundo os resultados do exame anátomo-patológico e tomografia computadorizada quanto à localização da lesão (superficial ou não superficial).

\begin{tabular}{lcc}
\hline & \multicolumn{2}{c}{ Localização } \\
\hline Tomografia computadorizada & Superficial & Profunda \\
Superficial & 1 & 1 \\
Profunda & 4 & 9 \\
\hline
\end{tabular}

sensibilidade de $40 \%$ e especificidade de $88,6 \%$ (Tabela 4).

Dos 15 exames de tomografia computadorizada, a consistência sólida das neoplasias foi demonstrada corretamente em 13 casos, com uma sensibilidade de $86,7 \%$ e especificidade de $0 \%$, e para cística em nenhum caso, com sensibilidade de $0 \%$ e especificidade de $86,7 \%$ (Tabela 5). Quanto a localização no lobo superficial, demonstrou corretamente em um caso, sensibilidade de $20 \%$ e especificidade de $90 \%$, e para o lobo profundo em nove casos, sensibilidade de $90 \%$ e specificidade de 20\% (Tabela 6). 


\section{DISCUSSÃO}

Em virtude da glândula parótida ser facilmente acessível à palpação, muitas condições patológicas são diagnosticadas clinicamente. Entretanto, é comum realizar-se pelo menos um método de diagnóstico por imagem na avaliação pré-operatória das neoplasias, para confirmação da localização em lobo superficial ou profundo, consistência e extensão. Atualmente, os métodos de diagnóstico por imagem mais usados são a ultra-sonografia de alta resolução com auxílio do "Doppler" colorido e TC, cada um com uma indicação e complementares entre si. Nosso estudo foi comparar dados de imagem e achados cirúrgicos de 56 pacientes com neoplasias da glândula parótida, para determinar o valor dos métodos diagnósticos por imagem na avaliação da extensão dessas lesões.

Apesar da US ter sido recomendada como método diagnóstico de investigação inicial na avaliação de massas da glândula parótida ${ }^{6,9}$, alguns autores acham o método inócuo, determinando somente a estrutura sólida ou cística do tumor ${ }^{4,10}$.

Para outros, a TC é o método de escolha, mesmo sem o emprego de contraste, nos tumores parotídeos ${ }^{3}$, ocorrendo eventualmente a necessidade do uso do contraste ${ }^{11}$. Em nosso trabalho, observamos que a US obteve êxito na demonstração de consistência cística ou sólida das neoplasias em 37 dos 45 exames (82,2\% dos casos). Nós entendemos que a TC sem contraste poderia ser usada, se necessário, como método de rastreamento da região parotídea, após a ultra-sonografia, mas deixa a desejar na qualidade da imagem em relação à delimitação dos vasos, diferenciação de linfonodomegalias, além do tipo de vascularização das neoplasias.

Em todos os nossos casos, foi usado contraste iodado endovenoso e os achados foram equivalentes aos achados cirúrgicos em 13 dos 15 casos para os tumores sólidos e falhou nos tumores císticos. Quanto à localização em relação à extensão no lobo profundo, foi coincidente com os achados cirúrgicos em nove casos e no lobo superficial em apenas um caso.

Entretanto, é inquestionável que há um aumento na eficácia da avaliação da doença pré-tratamento, e se, adequadamente usadas, as técnicas diagnósticas podem ajudar o cirurgião no estadiamento da doença e planejamento cirúrgico, melhorando o prognóstico ${ }^{7}$. Quando ocorre invasão do lobo profundo e do espaço parafaríngeo, o auxílio da imagem e exame citológico pós punção com agulha fina minimizam "surpresas" intra-operatórias, deixando claro porém que nem todas as lesões desta glândula requerem métodos de imagem $^{12,13}$.

Em nossos achados, observamos que a US colaborou na avaliação de lesões do lobo superficial e deixa a desejar nas do lobo profundo, devido à interposição do ramo da mandíbula, onde a TC mostrou eficiência.

Quanto à eficácia da US no diagnóstico das neoplasias da glândula parótida, considerando-se que $90 \%$ das neoplasias desta glândula originam do lobo superficial, a US de alta resolução apresenta alta sensibilidade $^{14}$. Nosso trabalho confirma esta afirmação, onde obtivemos resultados semelhantes para as neoplasias do lobo superficial, em 91,2 \% dos exames. Em dois casos a TC não demonstrou nenhuma alteração da glândula parótida. Em alguns casos a densidade do tecido das neoplasias da parótida pode ser similar ao do parênquima normal e o computador não consegue detectar ${ }^{15,16}$.

Com relação aos nossos resultados e aos da literatura, podemos inferir que a US deve ser usada como método diagnóstico inicial para neoplasias, e se uma lesão não for bem definida, então faz-se TC. Diante do exposto, em virtude da heterogeneidade da amostra, pode-se afirmar que é necessário um estudo prospectivo controlado, comparando esses métodos de diagnóstico por imagem, para que se possa estabelecer algoritmos diagnósticos, visando à melhor avaliação desses métodos e sua contribuição para o diagnóstico pré-operatório dos tumores da glândula parótida.

Em síntese, a US evidenciou bem as lesões sólidas do lobo superficial e falhou nas lesões císticas e na demonstração do lobo profundo, enquanto que a TC evidenciou bem as lesões do lobo profundo da glândula parótida, falhando nas lesões císticas no lobo superficial.

Podemos concluir que a US associada à TC, auxiliam no diagnóstico pré-operatório da consistência e extensão dos tumores da glândula parótida. 


\begin{abstract}
Background: The aim of this work was to evaluate the real value of ultrasonography (US) and computed tomography $(C T)$ in the surgical planning of neoplasias of the parotid gland. Methods: Retrospective study of 56 patients with neoplasias of the parotid gland (43 benigns and 13 malignants) from Head and Neck/Otorhinolaryngology and Image Departments of Hospital Heliopolis, Hosphel, São Paulo (1991 to 1999), comparing US and TC with histological findings. Results: From 45 US findings, a solid consistance was diagnosed in 33 cases (sensitivity of $89 \%$ and specificity of 50\%), and a cystic consistance in four cases (sensitivity and specificity of 50\%). Regarding the site, the superficial lobe was affected in 31 cases (sensitivity of $91.2 \%$ and specificity of $36.5 \%$ ), and the deep lobe in four cases (sensitivity of $40 \%$ and specificity of $88.6 \%$ ). For 15 CT procedures, the solid consistance was diagnosed in 13 cases (sensitivity of $86.7 \%$ and specificity of $0 \%$ ), and the cystic one in none (sensitivity of $0 \%$ and specificity of $86.7 \%$ ). Regarding the site, the superficial lobe, there was coincidence in one case (sensitivity of $20 \%$ and specificity of 90\%), and for deep lobe in nine cases (sensitivity of $90 \%$ and specificity of 20\%). Conclusions: 1 . US was a better choice for solid lesions of the superficial lobe but was not effective for deep lobe. 2. CT was better for lesions of the deep lobe, but ineffective for superficial lobe cystic lesions.
\end{abstract}

Key Words: Ultrasonography; Computed tomography; Patient care planning; Parotid gland; Neoplasm; Retrospective studies.

\section{REFERÊNCIAS}

1. Rasmussen SN, Christiansen NJ, Jorgensen JS, et al. - Differentiation between cystic and solid thyroid nodules by ultrasonic examination. An estimation of the value of ultrasonic examination as a supplement to isotope scanning. A preliminary communication. Acta Chir Scand, 1971, 137(4):331-333.

2. Hounsfield GN - Computerized transverse axial scanning (tomography): 1. Description of system. Br J Radiol, 1973,46(552):1016-1022.

3. Golding S - Computed tomography in the diagnosis of parotid gland tumours. Br J Radiol, 1982, 55(651):182-188.

4. Marins JLC - Cisto de parótida. Há necessidade de se usar 3 métodos de imagem para o diagnóstico? Arq Inst Penido Burnier, 1981, 26(1):78-80.

5. Whyte AM, Hourihan MD - The diagnosis of tumours involving the parapharyngeal space by computed tomography. Br J Radiol, 1989, 62(738): 526-531.

6. Isaza M, Ikezoe J, Morimoto S, et al. - Computed tomography and ultrasonography in parotid tumors. Acta Radiol, 1989, 30(1):11-15.

7. Noyek AM, Kassel EE, Chapnik JS, et al. - Parotid gland and parapharyngeal space imaging: the surgical significance. Isr J Med Sci, 1992, 28(3-4):193-197.

8. Eracleous E, Kallis S, Tziakouri C, et al. - Sonography, CT, CT sialography, MRI and MRI sialography in investigation of the facial nerve and the differentiation between deep and superficial parotid lesions. Neuroradiology, 1997, 39(7): 506-511.
9. Whyte AM, Byrne JV - A comparison of computed tomography and ultrasound in the assessment of parotid masses. Clin Radiol, 1987, 38(4): 339-343.

10. Peiró Escrivá J, Torregrosa Huguet T, Teruel Escrivá S, et al. - Tumores de la glándula parórida: semiología ecográfica. Rev Mex Radiol, 1985, 39(3): 117-121.

11. McGahan JP, Walter JP, Bernstein L- Evaluation of the parotid gland. Comparison of sialography, noncontrast computed tomography, and CT sialography. Radiology, 1984, 152(2):453-458.

12. Marins JLC, Prando A, Pereira RM, et al. - Correlação entre sialografia, ultra-sonography e TC no diagnóstico das lesões benignas da parótida. Radiol Bras, 1982, 15(1): 64-67.

13. Noyek AM, Kassel EE - Contemporary imaging of parotid gland and parapharyngeal space. Can J Surg, 1987,30(6): 395-397.

14. Wittich GR, Scheible WF, Hajek PC-Ultrasonography of the salivary glands. Radiol Clin North Am, 1985, 23(1): 29-37.

15. Rice DH, Mancuso AA, Hanafee WN - Computerized tomography with simultaneous sialography in evaluating parotid tumors. Arch Otolaryngol, 1980, 106(8): 472-473.

16. Bradley MJ, Ahuja A, Metreweli C - Sonographic evaluation of the parotid ducts: its use in tumors localization. Br J Radiol, 1991, 64(768):1092-1095.

Endereço para correspondência:

Prof. Dr. Abrão Rapoport

Rua Iramaia, 136-Jd. Europa-CEP-01450-020-São Paulo

Telefax: (11) 273-8224

E-mail: cpgcp.hosphel@attglobal. 